\title{
INCORPORAÇÃO DE FIBRA ALIMENTAR EM PRODUTOS CÁRNEOS
}

\author{
Incorporation of dietary fiber in meat products
}

\author{
Ana Paula Burin Fruet I', Flávia Santi Stefanello', Mariana Santos da Silva², Jackeline Karsten Kirinus 3 , José \\ Laerte Nörnberg ${ }^{4}$, César Teixeira ${ }^{5}$, Andrea Cristina Dörr ${ }^{6}$
'Médicas Veterinárias, Alunas do Programa de Pós-Graduação em Ciência e Tecnologia de Alimentos da Universidade Federal de Santa Maria (UFSM).*Autor para correspondência: ap_burin@hotmail.com
${ }^{2}$ Acadêmica do curso de Farmácia (UFSM).
${ }^{3}$ Médica Veterinária, Professora do curso de Medicina Veterinária, Universidade do Oeste de Santa Catarina (UNOESC).
${ }^{4}$ Médico Veterinário, Professor Associado do Departamento de Tecnologia e Ciência dos Alimentos (UFSM). Zaragoza \\ ${ }^{5}$ Advogado e Mestre em Direito Ambiental pela Universidade de Zaragoza (Espanha). Doutorando em Direito Civil pela Universidade de \\ ${ }^{6}$ Economista, Professora Adjunto do Departamento de Ciências Econômicas (UFSM).
}

\begin{abstract}
Resumo
Fibra alimentar, ou fibra dietética, pode ser classificada, de acordo com sua funcionalidade, em solúvel e insolúvel. Fibras insolúveis apresentam porosidade, baixa densidade, capacidade de aumentar a produção fecal, devido à hidratação desta, e promover os movimentos intestinais regulares. Em contrapartida, a fração solúvel caracteriza-se por formar gel, atuar como emulsificante e sofrer fermentação. Em virtude da viscosidade e da fermentação, a fibra solúvel apresenta maior relevância à saúde, pois atua na redução dos níveis de colesterol sanguíneo, manutenção da glicemia, colabora com o sistema imunológico, previne doenças (entre elas o câncer) e contribui com a diminuição do esvaziamento gástrico. As fibras solúveis podem ser incorporadas em maior quantidade sem alterar a textura e o sabor dos alimentos quando comparadas com as frações insolúveis, neste sentido, vários estudos são conduzidos com intuito de avaliar a adição de diferentes fibras em produtos cárneos. Carnes e derivados são reconhecidos pela alta composição lipídica indesejada à saúde, tal atributo aliado às características reológicas e funcionais das fibras, permite a substituição de gordura por carboidratos não digestíveis. Destaca-se ainda vantagens como o aumento da capacidade de retenção de água, incremento da estabilidade de emulsão que auxilia o rendimento do produto, além da elaboração de um produto funcional sem modificar sua aceitabilidade.
\end{abstract}

Palavras - chave: alimento funcional, $\square$-glicana, carne, fibra dietética, produto enriquecido.

\begin{abstract}
Dietary fiber can be classified according to their functionality in soluble and insoluble. Insoluble dietary fibers have porosity, low density, ability to increase fecal bulk due to the hydration there of and to be higher and transit time enhances bowel. In contrast, the soluble fraction is characterized by forming gel, acting as an emulsifier and suffers fermentation. Due to the viscosity and fermentation, soluble fiber has greater relevance to health as it acts in reducing blood cholesterol levels, blood glucose control helps with the immune system, prevent diseases, among these cancer, and contributes to the decrease of hollowing stomach. Soluble fiber can be incorporated in a larger quantity without changing the texture and tastes of food when compared with the insoluble fractions, in this sense, several studies are conducted in order to assess the addition of fiber in meat products. Meat and meat products are recognized by high lipid composition unwanted health, such combined rheological and functional characteristics of the fibers, attribute allows the replacement of fat by non-digestible carbohydrates. Another highlight advantages such as increased water retention capacity, increased emulsion stability that helps product yield, besides the development of a functional product without changing its acceptability.

Keywords: functional food, $\square$-glucan, meat, dietary fiber, enriched product.
\end{abstract}




\section{INTRODUÇÃO}

O padrão alimentar dos seres humanos mudou para uma dieta em que os grãos refinados, carnes, gorduras e açúcares adicionados são amplamente consumidos, enquanto a ingestão de proteínais vegetais e fibras são reduzidas (KENDALL et al., 2010). Estas alterações, juntamente com o sedentarismo, trouxeram uma série de problemas relacionados à saúde do consumidor, destacando-se a obesidade, as doenças cardíacas, a diabetes tipo 2 e o câncer. Atualmente, com intuito de produzir alimentos saudáveis e que previnam tais alterações, busca-se desenvolver produtos funcionais conceituados como alimentos que demonstram benefícios fisiológicos à saúde, além da funcionalidade nutricional.

Dentre os produtos adicionados, fibras alimentares são pesquisadas pelos efeitos benéficos reconhecidos por colaborar com as propriedades sensoriais de alimentos. Apesar dos produtos cárneos serem reconhecidos pelo alto teor de lipídios, o processamento de carnes permite a adição de ingredientes e elaboração de derivados cárneos funcionais. Neste sentido, esta revisão retrata as características de fibra alimentar, com ênfase na importância para a saúde e sua aplicabilidade em produtos cárneos.

\section{BENEFÍCIOS DA FIBRA ALIMENTAR}

De acordo com a comissão do Codex Alimentarius, fibras dietéticas, também chamadas de fibras alimentares, são polímeros de carboidratos não digeridos, tampouco absorvidos no intestino delgado. A fibra dietética pode ser classificada nas seguintes categorias: polímeros de carboidratos comestíveis, inerentes aos alimentos que são consumidos; polímeros de carboidratos obtidos a partir de matéria-prima alimentar por meio de procedimentos enzimáticos, físicos ou químicos, os quais tenham mostrado efeito fisiológico benéfico à saúde ,através de meios científicos aceitos pelas autoridades competentes; ou polímeros de carboidratos sintéticos que apresentam efeito fisiológico benéfico à saúde, através de meios científicos aceitos pelas autoridades competentes (CUMMINGS et al., 2009; MANN; CUMMINGS, 2009; MENEZES et al., 2013).

Outros conceitos são utilizados para fibra dietética, segundo a American Association of Cereal Chemists (AACC), fibras são partes das plantas ou carboidratos análogos resistentes à digestão e absorção no intestino delgado de humanos, contudo são fermentados parcial ou completamente no intestino grosso. Estão incluídos neste grupo: polissacarídeos, oligossacarídeos, substâncias ligadas às fibras, como a lignina (MENEZES et al., 2013). Enquanto que Wismar et al. (2005) propuseram um conceito de fibras funcionais, sendo estas carboidratos não-digeríveis e fisiologicamente benéficos.

Evidencia-se que não existe um único conceito para fibra dietética, portanto, algumas características devem estar presentes na definição, tais como composição da fibra, resistência à digestão e absorção no intestino delgado, fermentação no intestino grosso e ainda demonstrar efeitos benéficos para a saúde (LUNN; BUTTRISS, 2007).

As fibras alimentares são amplamente estudadas em decorrência de seus benefícios, entre eles: a redução de colesterol sanguíneo, melhorias na função do intestino grosso e diminuição da glicemia pós-prandial (colaborando desta forma para a prevenção ou redução de doenças intestinais), diminuição do risco de doença cardíaca coronária e diabetes do tipo 2 (KENDALL et al., 2010; MANN; CUMMINGS, 2009; MUDGIL; BARAK, 2013).

Além disso, as fibras também colaboram para as propriedades reológicas de produtos (MUDGIL; BARAK, 2013) através de características como a solubilidade, viscosidade, formação de gel, capacidade de retenção de água e aumento de volume através de associação entre moléculas (CUMMINGS; STEPHEN, 2007; MUDGIL; BARAK, 2013). Dentre as propriedades, a solubilidade das fibras é relevante, principalmente, por definir os efeitos tecnológicos e fisiológicos.

Com base nesta característica, os carboidratos não digestíveis podem ser classificados como solúveis e insolúveis (Figura 1), sendo que fibras dietéticas solúveis formam uma solução quando misturado com água e o mesmo não ocorre com os componentes insolúveis (ELLEUCH et al., 2011). Os autores esclarecem que a condição fisiológica e efeitos físico-químicos das fibras dietéticas dependem da quantidade de fibra, especialmente, no que se refere às frações solúveis e insolúveis. 


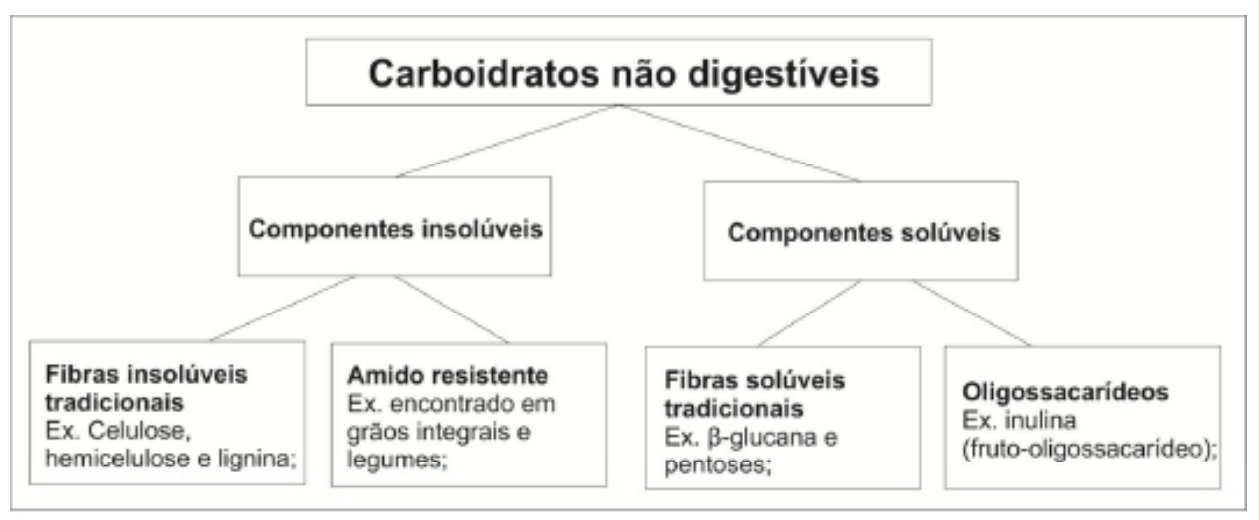

Figura 1. Classificação de carboidratos não digestíveis com ênfase na solubilidade.

Fonte: Lunn e Buttriss (2007).

\section{FIBRAS INSOLÚVEIS}

Compõe esta categoria a celulose, hemicelulose, lignina e amido resistente (ELLEUCH et al., 2011; MUDGIL; BARAK, 2013), podem ser encontrados em alimentos de origem vegetal pelo fato de a parede celular conter fibras não digeríveis no trato gastrointestinal humano. Legumes, cereais e alimentos integrais são fontes de fibra dietética (LUNN; BUTTRISS, 2007).

Fibras insolúveis são caracterizadas pela porosidade, baixa densidade, capacidade para aumentar a produção fecal e diminuir o tempo de trânsito intestinal (ELLEUCH et al., 2011; MIRA et al., 2009; MANN; CUMMINGS, 2009). Para que fibras insolúveis provoquem redução do colesterol, é necessário ser adicionado em grandes quantidades, bem como ser rapidamente fermentável. Em contrapartida, a inclusão de componentes que fermentam lentamente, como amidos resistentes, pode trazer melhorias na função do intestino (LUNN; BUTTRISS, 2007).

A celulose e algumas hemiceluloses são insolúveis em água, apresentam capacidade de se ligar à água e hidratar as fezes, o que aumenta o volume fecal e, assim, promove movimentos intestinais regulares. Embora os seres humanos não sejam capazes de digerir estes carboidratos, ocorre uma digestão parcial no intestino grosso pela microflora, tal fermentação natural no cólon produz uma quantidade significativa de ácidos graxos de cadeia curta, benéficos para as células intestinais (MUDGIL; BARAK, 2013).

\section{FIBRAS SOLÚVEIS}

Fibras alimentares solúveis incluem subs- tâncias pécticas, gomas, mucilagens, alginatos, $\beta$-glicanas (ELLEUCH et al., 2011) e arabinoxilanas (IZYDORCZYK; DEXTER, 2008). As algas e subprodutos de frutos parecem ser fontes ricas em fibras solúveis, seguida de fruta, legumes e cereais (CUMMINGS; STEPHEN, 2007; ELLEUCH et al., 2011).

Quando avaliadas as propriedades funcionais de fibras, observa-se que a fração solúvel demonstra uma maior capacidade para proporcionar a viscosidade, formar géis e atuar como emulsificante, sem alterar a textura e o gosto do alimento, sendo assim, apresenta maior facilidade para ser incorporada em alimentos processados e bebidas (ELLEUCH et al., 2011). Quanto ao grau de fermentação, fibras solúveis são altamente fermentadas quando comparadas com fibras insolúveis, esta propriedade, juntamente com a alta viscosidade, pode ter um papel importante em alguns efeitos fisiológicos da fibra (MUDGIL; BARAK, 2013). Também são especialmente importantes na redução dos níveis de colesterol no sangue e índice glicêmico (BAIK; ULLRICH, 2008; BARSANTI et al., 2011; DICKIN et al., 2011; WOOD, 2007), além de contribuir com o sistema imunológico, prevenir doenças como o câncer (BARSANTI et al., 2011; YOON et al., 2013) e retardar o esvaziamento gástrico (MIRA et al., 2009).

A aplicabilidade de fibras solúveis como ingrediente alimentar é amplamente considerada com duplo propósito: aumentar o teor de fibra em alimentos para gerar benefícios à saúde e melhorar as propriedades funcionais ou tecnológicas de produtos (IZYDORCZYK; DEXTER, 2008; EL KHOURY et al., 2012; MUDGIL; BARAK, 2013).

Fibras dietéticas podem ser usadas em produtos alimentares processados, não só para incrementar o teor de carboidrato não digestível, 
mas também para colaborar com a viscosidade, a textura, as características organolépticas e a vida de prateleira de produtos alimentares. Muitos subprodutos da indústria de alimentos são ricos em fibras e podem servir como fonte de fibra para incorporação em alimentos processados. São exemplos de subprodutos os resíduos de frutas e indústria de cereais que podem ser utilizados para substituição de farinha e gordura, melhora da retenção de água e emulsão de óleo. Deve-se destacar que existe uma variação de incorporação máxima de fibra em diferentes produtos, pois excesso de fibra pode trazer alterações indesejáveis na cor e textura dos alimentos (MUDGIL; BARAK, 2013).

Outra característica benéfica de fibras altamente viscosas, como $\beta$-glicanas, hidrocoloides e pectinas, é a influência nos níveis lipídicos do sangue (MUDGIL; BARAK, 2013). Sabe-se que fibras solúveis diminuem níveis de colesterol sanguíneo, em decorrência da capacidade de se ligar aos ácidos biliares sintetizados no fígado a partir do colesterol, impedindo-os de serem reabsorvidos, consequentemente há um aumento da conversão do colesterol em ácidos biliares e redução dos níveis de colesterol no sangue. Além deste mecanismo, também é proposto que a resposta glicêmica reduzida conduz um estímulo menor para a síntese do colesterol hepático e a maior quantidade de propionato, produzido durante a fermentação, tem um grande potencial para redução do colesterol (GUNNESS; GIDLEY, 2010; KIM; WHITE, 2010). Kaczmarczyk et al. (2012) relatam ainda que existe um aumento da expressão de enzimas limitantes da síntese de colesterol, a HMG-CoA redutase, que diminui a síntese de colesterol e aumenta a excreção deste através da vesícula biliar.

Pesquisadores estabeleceram papel significativo de $\beta$-glicanas sob o sistema imunológico em relação ao tratamento do câncer, a imunidade à infecção e restauração de medula óssea danificada. Como agente imunomodulador, $\beta$-glicana atua através da ativação de células do sistema imunológico inato, como macrófagos, células dendríticas e granulócitos. Esta ativação desencadeia as respostas das células imunes adaptativas, resultando na inibição do crescimento de tumores e metástase (YOON et al., 2013).

A interação entre fibras solúveis e outros componentes dos alimentos também apresenta potencial de influenciar na digestibilidade do amido, fibras solúveis podem reduzir a glicemia, diminuindo a absorção de carboidratos no intestino através de aumento da viscosidade (KACZMARCZYK et al., 2012). Da mesma forma, Regand et al. (2011) demonstraram que a inibição de digestibilidade do amido por $\beta$-glicana está relacionado com a viscosidade. Ainda não está claro se a redução é uma consequência de alterações nas propriedades físicas do amido, por limitação de água disponível para a hidratação, ou se a formação de um extrato concentrado de $\beta$-glicana inibe enzimas que se ligariam aos grânulos de amido parcialmente gelatinizados.

Dietas ricas em fibras solúveis levam ao aumento da saciedade e diminuição na ingestão voluntária de alimentos, isso devido ao retardamento do esvaziamento gástrico e a absorção de glicose em virtude da viscosidade no conteúdo luminal do trato gastrointestinal (BAIK; ULLRICH, 2008; MIRA et al., 2009).

Apesar de muitos estudos demonstrarem efeitos benéficos das fibras solúveis, algumas pesquisas não conseguem retratar uma ligação entre a ingestão de fibras viscosas e risco de doença coronariana. $\mathrm{O}$ achado pode ser atribuído ao fato de que a ingestão de fibra solúvel pode ser muito baixa em países ocidentais e, portanto, os efeitos não podem ser efetivamente avaliados (KENDALL et al., 2009). Sendo assim, resultados de uma meta-análise estimou que é necessário o consumo de $3 \mathrm{~g} /$ dia de $\beta$-glicana, proveniente de aveia ou cevada, para diminuir o colesterol sanguíneo, no entanto o efeito sobre a glicose sanguínea ainda é inconclusiva, com grande heterogeneidade, e requer estudos de pesquisa clínica mais prolongados (TIWARI; CUMMINS, 2011).

Resultados de estudo com voluntários humanos ilustram que a concentração de $\beta$-glicana é fundamental para determinar a sua utilidade para a saúde humana, portanto, a magnitude do benefício para a saúde será determinada pela genética e fatores ambientais que controlam a concentração de $\beta$-glicana no grão de cevada, por exemplo, e, portanto, variações podem afetar a atividade biológica de grãos (DICKIN et al., 2011).

Dickin et al. (2011) ainda retratam a necessidade de desenvolver variedades de cevada destinadas à indústria alimentar, ou seja, cultivares de cevada nua (nuda) que contenham um nível consistente de $\beta$-glicana. Pois, atualmente, a produção é destinada principalmente à indústria de fabricação cervejeira (DE MORI, 2007) ou para produção de forragem aos animais (KURTZ, 2012). Um alto teor de $\beta$-glicana na cevada é um problema na filtração de cerveja, aumenta a viscosidade do mosto e reduz o rendimento do produto acabado (ABUGALIEVA et al., 2012). 


\section{ADIÇÃO DE FIBRA ALIMENTAR EM PRO- DUTOS CÁRNEOS}

O aumento do consumo de produtos cárneos, aliado à necessidade de produzir alimentos saudáveis com boa aceitabilidade, incrementou os estudos sobre o uso de fibras. Assim, a incorporação de componentes funcionais em derivados cárneos oferece benefícios adicionais à saúde, além da nutrição básica, o que os torna reconhecidos como alimentos funcionais (HAO; BETA, 2012). Tais produtos enriquecidos são mais saudáveis quando comparados com os convencionais, no entanto a produção de alimentos funcionais limita-se, uma vez que pode alterar as características sensoriais (DECKER; PARK, 2010; MEHTA et al., 2013). Alguns trabalhos têm demonstrado que a adição de fibra em salsichas (CHOI et al., 2009) e em hambúrgueres (SAYAGO-AYERDI, 2009), em níveis de $2 \%$, pode ser realizada sem impacto negativo na qualidade sensorial. Outros estudos indicam que a adição de mais de $10 \%$ de fibra alimentar não causa impacto significativo na análise sensorial de hambúrgueres (PINHO et al., 2011), empanados de carne (PIÑERO et al., 2008) e em salsichas (CHOE et al., 2013).

Destaca-se que para a legislação brasileira, de acordo com a Portaria 27 (BRASIL, 1998), define-se produto rico em fibras quando o teor mínimo de fibra alimentar for de $6 \mathrm{~g}$ em $100 \mathrm{~g}$ de produto sólido $(3 \mathrm{~g} / 100 \mathrm{ml}$ para produtos líquidos), enquanto que produto fonte de fibra deve apresentar teor mínimo de $3 \mathrm{~g} / 100 \mathrm{~g}$ (1,5g/100 ml para líquidos). É válido pontuar a recomendação de consumo médio de 21-38g/dia de fibra alimentar. Sendo que os nutricionistas e especialistas em dieta sugerem que $20-30 \%$ da ingestão diária de fibra deve ser composta por fibras solúveis (ELLEUCH et al., 2011; MEHTA et al., 2013).

Devido à divergência a respeito da ação de alimentos ricos em gorduras, como carnes, no aumento dos depósitos de gordura corporal, Cristofoletti et al. (2013) avaliaram a relação entre o consumo de embutidos cárneos e a obesidade. Em tal estudo foi observado uma associação positiva entre os dois fatores. Fibra alimentar pode substituir a gordura de produtos cárneos, gerando baixo teor de lipídios e aumento de fibra (BREWER, 2012; PIÑERO et al., 2008; ZHANG et al., 2010), além de outras vantagens reológicas como o aumento da capacidade de retenção de água, incremento da estabilidade de emulsão, o que resulta em um produto com maior rendimento após cozimento e melhora na estabilidade oxidativa quando as fontes de fibras estão relacionadas com antioxidantes fenólicos (CHOI et al., 2009; MEHTA et al., 2013).

Choe et al. (2013) relatam que ao adicionar diferentes níveis de fibra alimentar em salsichas foi observado uma relação inversamente proporcional com a perda por cozimento, tal fato é explicado pela alta capacidade da fibra reter água e gordura.

$\mathrm{O}$ uso de fibra alimentar e antioxidantes naturais são dois fatores dietéticos envolvidos na promoção da saúde, neste sentido, Sayago-Ayerdi et al. (2009) adicionaram bagaço de uva em hambúrgueres e observaram uma inibição da oxidação e estabilidade lipídica, além de uma elevação nos efeitos benéficos à saúde, sem interferir na aceitabilidade do alimento. Outra abordagem promissora proposta por Saura-Calixto (2011) é o estudo de antioxidantes ligados à fibra alimentar, o autor relata que cerca de $50 \%$ do total de antioxidantes da dieta, principalmente polifenóis, atingem o intestino ligado às fibras, contribuindo desta forma para ação antioxidante no local.

\section{CONCLUSÃO}

Fibra alimentar é uma fração de carboidrato relevante à saúde em decorrência de suas características determinadas, principalmente, através da solubilidade. Fibras insolúveis colaboram com a formação fecal e função intestinal, enquanto que fibras solúveis apresentam efeitos acentuados à saúde, tais como o controle da glicemia, a diminuição do colesterol, além da prevenção do câncer e contribuição com a sensação de saciedade. Esta fração viscosa é um ingrediente funcional que permite ser incorporado em produtos cárneos, devido às suas peculiaridades reológicas, produzindo, assim, alimentos enriquecidos sem prejudicar os atributos sensoriais e a aceitabilidade de produto.

\section{REFERÊNCIAS}

ABUGALIEVA, A.I. et al. Significance of $\beta$ Glucan in Barley Grain during Breeding for Brewing and Nutritional Properties. Russian Agricultural Sciences, v.38, n.2, p.89-93, 2012.

BAIK, B.K.; ULLRICH, S.E. Barley for food: Characteristics, improvement, and renewed interest. Journal of Cereal Science, v.48, p.233-242, 2008.

BARSANTI, L. Chemistry, physico-chemistry and applications linked to biological activities of 
$\beta$-glucans. Natural Product Reports, v.28, p.457-466, 2011.

BRASIL. Agência Nacional de Vigilância Sanitária. Portaria $\mathrm{n}^{\circ} 27$. Aprova o Regulamento Técnico referente à Informação Nutricional Complementar (declarações relacionadas ao conteúdo de nutrientes), constantes do anexo desta Portaria. Diário Oficial da União, Brasília, 1998.

BREWER, M.S. Reducing the fat content in ground beef without sacrificing quality: A review. Meat Science, v.91, p.385-395, 2012.

CHOE, J.H. et al. Quality of frankfurter-type sausages with added pig skin and wheat fiber mixture as fat replacers. Meat Science, v.93, p.849-854, 2013.

CHOI, Y.S. Characteristics of low-fat meat emulsion systems with pork fat replaced by vegetable oils and rice bran fiber. Meat Science, v.82, p.266-271, 2009.

CRISTOFOLETTI, M.F. et al. Associação entre consumo de alimentos embutidos e obesidade em um estudo de base populacional de nipo-brasileiros. Arquivos Brasileiros de Endocrinologia e Metabologia, v.57, n.6, p.464-472 2013.

CUMMINGS, J.H.; STEPHEN, A.M. Carbohydrate terminology and classification. European Journal of Clinical Nutrition, v.61, S5-S18, 2007.

CUMMINGS, J.H. et al. Dietary fibre: a agreed definition. Lancet, v.373, n.9661, p.365-366, 2009.

DECKER, E.A.; PARK, Y. Healthier meat products as functional foods. Meat Science, v.86, p.49-55, 2010.

DE MORI, C. et al. Dinâmica da produção de cevada no Brasil no período de 1975 a 2003. Passo Fundo: Embrapa Trigo, Boletim de Pesquisa e Desenvolvimento Online. v.37, 2007. 33 p. Disponível em: <http://www.cnpt.embrapa.br/biblio/ bp/p_bp37.htm>. Acesso em: 15 ago. 2013.

DICKIN, E. et al. Effect of genotype, environment and agronomic management on $\beta$-glucan concentration of naked barley grain intended for health food use. Journal of Cereal Science, v.54, n.1, p.44-52, 2011.

ELLEUCH, M. et al. Dietary fibre and fibre-rich by-products of food processing: Characterization, tech- nological functionality and commercial applications: A review. Food Chemistry, v.124, p.411-421, 2011.

EL KHOURY, D. et al. Beta Glucan: Health Benefits in Obesity and Metabolic Syndrome. Journal of Nutrition and Metabolism, v.1, p.1-29, 2012.

GUNNESS, P.; GIDLEY, M.J. Mechanisms underlying the cholesterol-lowering properties of soluble dietary fibre polysaccharides. Food \& function, v.1, n.2, p.149-155, 2010.

HAO, M.; BETA, T. Development of Chinese steamed bread enriched in bioactive compounds from barley hull and flaxseed hull extracts. Food Chemistry, v.133, p.1320-1325, 2012.

IZYDORCZYK M.S.; DEXTER J.E. Barley b-glucans and arabinoxylans: Molecular structure, physicochemical properties, and uses in food products-a Review. Food Research International, v.41, p.850868,2008 .

KACZMARCZYK, M.M.; MILLER, M.J.; FREUND, G.G. The health benefits of dietary fiber: Beyond the usual suspects of type 2 diabetes mellitus, cardiovascular disease and colon cancer. Metabolism clinical and experimental, v.61, p.1058-1066, 2012.

KENDALL, C.W.C.; ESFAHANI, A.; JENKINS, D.J.A.The link between dietary fibre and human health. Food Hydrocolloids, v.24, p.42-48, 2010.

KIM, H.J.; WHITE, P.J. In vitro bile-acid binding and fermentation of high, medium, and low molecular weight $\beta$-glucan. Journal of agricultural and food chemistry, v.58, n.1, p.628-634, 2010.

KURTZ, P. Quando a cevada é a escolha certa. Embrapa. 2012. Disponível em: <http://www.cnpt. embrapa.br/noticias/2012/not1236.htm>. Acesso em: 03 ago. 2013.

LUNN, J.; BUTTRISS, J.L. Carbohydrates and dietary fibre. British Nutrition Foundation Nutrition Bulletin, v.32, p. 21-64, 2007.

MANN, J.I.; CUMMINGS, J.H. Possible implications for health of the different definitions of dietary fibre. Nutrition, Metabolism \& Cardiovascular Diseases, v.19, p.226-229, 2009.

MEHTA, N. et al. Novel trends in development of dietary fiber rich meat products - a critical review. 
Journal of Food Science and Technology, p.1-15, 2013.

MENEZES, E.W. et al. Codex dietary fibre definition - Justification for inclusion of carbohydrates from 3 to 9 degrees of polymerization. Food Chemistry, v.140, p.581-585, 2013.

MIRA, G.S.; GRAF, H.; CÂNDIDO, L.M. Visão retrospectiva em fibras alimentares com ênfase em beta-glucanas no tratamento do diabetes. Brazilian Journal of Pharmaceutical Sciences, v.45, n.1, p.1120, 2009.

MUDGIL, D.; BARAK, S. Composition, properties and health benefits of indigestible carbohydrate polymers as dietary fiber: A review. International Journal of Biological Macromolecules, v.61, p.1-6, 2013.

PIÑERO, M.P. et al. Effect of oat's soluble fibre (b-glucan) as a fat replacer on physical, chemical, microbiological and sensory properties of low-fat beef patties. Meat Science, v.80, p.675-680, 2008.

PINHO, L.X. et al. The use of cashew apple residue as source of fiber in low fat hamburgers. Ciência e Tecnologia dos Alimentos, v.31, n.4, p.941-945, 2011.

REGAND, A. et al. The molecular weight, solubility and viscosity of oat beta-glucan affect human glycemic response by modifying starch digestibility. Food Chemistry, v.129, p.297-304, 2011.

SAURA-CALIXTO, F. Dietary fiber as a carrier of dietary antioxidants: an essential physiological function. Journal of agricultural and food chemistry, v. 59, n. 1, p. 43-49, 2011.

SAYAGO-AYRDI, S.G.; BRENES, A.; GOÑI, I. Effect of grape antioxidant dietary fiber on the lipid oxidation of raw and cooked chicken hamburgers. LWT - Food Science and Technology, v.42, p.971976, 2009.

TIWARI, U.; CUMMINS E. Meta-analysis of the effect of b-glucan intake on blood cholesterol and glucose levels. Nutrition, v.27, p.1008-1016, 2011.

ZHANG, W. et al. Improving functional value of meat products. Meat Science, v.86, p.15-31, 2010.

YOON, T.J.; SUSHRUTA, K.; HO, L.K. The effects of $\beta$-glucans on cancer metastasis. Anti-cancer Agents in Medicinal Chemistry, v.13, n.5, p.699-708,
2013.

WISMAR, R. et al. Dietary fibers as immune regulatory compounds in health and disease. Annals of the Ney York Academy of Sciences, v.1190, p.70-85, 2010.

WOOD, P. Cereal b-glucans in diet and health. Journal of Cereal Science, v.46, p.230-238, 2007. 